\title{
Carriage of antibiotic-resistant Enterobacteriaceae in hospitalised children in tertiary hospitals in Harare, Zimbabwe
}

Marcelyn T. Magwenzi ${ }^{*}$ (D), Muchaneta Gudza-Mugabe², Hilda A. Mujuru ${ }^{3,4}$, Mutsa Dangarembizi-Bwakura ${ }^{3,5}$, Valerie Robertson ${ }^{1,6}$ and Alexander M. Aiken ${ }^{6,7}$

\begin{abstract}
Background: Extended-spectrum $\beta$-lactamase-producing and gentamicin resistant Enterobacteriaceae are increasingly recognised as a major cause of infection in low-income countries. We assessed the prevalence of gastrointestinal carriage of these bacteria in hospitalised children in Harare, Zimbabwe.

Methods: We conducted a cohort study in paediatric inpatients at two tertiary-referral hospitals between May and July 2015. Rectal swabs and faecal samples were collected within $24 \mathrm{~h}$ of admission and further follow-up samples were collected on alternate days during hospitalization. Disc-based, selective and enrichment methods were used to detect carriage of these two forms of resistance. Standard methods were used to confirm resistance status and determine the susceptibility of resistant isolates to other commonly-used antibiotics.

Results: One hundred and sixty four paediatric inpatient admissions (median age $=1.0$ year, $I Q R=0.2-2$.2years) were enrolled, and an average of 1.9 faecal samples per patient were collected. On admission, 68/164 (41\%) patients had both ESBL and gentamicin-resistant Enterobacteriaceae detected, 18 (11\%) had ESBL only, 17 (10\%) had gentamicin resistance only and 61 (37\%) had negative screening for both forms of resistance. During hospitalisation, 32/164 (20\%) patients were found to have a type of resistant organism which was not present in their admission sample. We found that faecal samples and use of a selective enrichment broth enhanced the detection of resistant organisms. Amongst resistant bacteria isolated, there were high levels of resistance to ciprofloxacin and chloramphenicol, but not ertapenem.

Conclusions: More than half of children had enteric carriage of a clinically-relevant form of antibiotic resistance on admission to public-sector hospitals in urban Zimbabwe. Additionally, a fifth of children acquired a further form of resistance during hospitalisation. Urgent action is needed to tackle the spread of antibiotic resistant enteric bacteria in African hospitals.
\end{abstract}

Keywords: Enterobacteriaceae, ESBL, Antibiotic resistance, Colonization, Children

\section{Background}

Clinically significant levels of resistance to various antibiotic classes have developed in most Gram-negative bacteria, though there is marked geographic variation in the prevalence of resistance [1]. Historically, many forms of antibiotic resistant organisms emerged in and were disseminated from healthcare facilities in high-income

\footnotetext{
* Correspondence: mmagwenzi@yahoo.com

${ }^{1}$ College of Health Sciences, Department of Medical Microbiology, University of Zimbabwe, PO Box A178, Harare, Zimbabwe

Full list of author information is available at the end of the article
}

settings, but in more recent times, hospitals in low and middle-income settings appear to have been the origin and amplifiers of several forms of resistance [2-4]. Weak antibiotic stewardship and limited infection control resources promote the spread of antibiotic resistant organisms in hospitals, especially where there are no microbiological diagnostic services for the identification of resistance [5].

In the family of Enterobacteriaceae, organisms such as Escherichia coli and Klebsiella pneumoniae can produce extended-spectrum $\beta$-lactamase (ESBL) enzymes, which 
confer resistance to third generation cephalosporins and most penicillins but are inhibited by agents such as clavulanate. These types of resistant bacteria are increasingly implicated as causes of both community- and hospital-acquired infections [6, 7] and are associated with higher levels of mortality than non-ESBL producers [8]. Across sub-Saharan Africa, a limited number of studies have described high levels of ESBL faecal carriage in children in association with hospital facilities [9-13]. Gentamicin is an aminoglycoside antibiotic that has been used for the treatment of Gram-negative infections for more than 40 years [14]. Its low-cost and broad spectrum of activity means that it is widely used for treatment of severe infections in many low-income countries.

The gastrointestinal tract is the principal ecological niche for Enterobacteriaceae. In this environment, interand intra-species exchange of resistance genes can occur and under appropriate selective pressure, resistant species can rapidly emerge and dominate [15]. While faecal carriage of antibiotic resistant bacteria is not an immediate threat to a healthy individual, it poses two risksfirstly, when auto-infection of a sterile body-site subsequently occurs (for example, hospital-acquired bacteraemia [16]) treatment is substantially more difficult and secondly, there may be transmission of the resistant organism to other individuals [17].

In order to describe levels of carriage of Gram-negative antibiotic resistance amongst hospitalised children in Zimbabwe, we aimed to measure 1) the prevalence of carriage of ESBL producing and gentamicin-resistant enteric bacteria in paediatric patients at the time of admission to hospital and 2) the risk of acquisition for the same types of bacteria during the corresponding time of hospitalization. We also sought to evaluate different methods for screening for the presence of these resistant organisms.

\section{Methods \\ Study design}

This was a prospective observational cohort study conducted in paediatric wards at two tertiary government hospitals in Harare, Zimbabwe between May and July 2015. Paediatric patients were recruited within $24 \mathrm{~h}$ of admission to hospital and remained in the study until discharge from hospital or death. All paediatric patients were eligible for inclusion in the study regardless of their presenting illness, provided that they remained in hospital for at least one night. These hospitals had paediatric wards divided into cubicles of 6 to 8 beds and limited isolation rooms. Each cubicle or room had a single hand-wash basin; alcohol-based hand sanitizers were not consistently available. There was no established practice of screening for carriage of resistant organisms in these wards at the time of this study.

\section{Screening for ESBL and gentamicin resistance}

Two rectal swabs and/or a stool sample were collected within $24 \mathrm{~h}$ of admission. Further similar samples were collected three times per week for all participants. The swabs and stool samples were directly inoculated onto a range of screening plates in the Department of Medical Microbiology laboratory, University of Zimbabwe, as follows:

1) MacConkey agar without salt (Oxoid, UK) plates with either a Cefpodoxime $(10 \mu \mathrm{g})$ or a Gentamicin $(10 \mu \mathrm{g})$ disc placed on the plate. The plates were incubated at $37^{\circ} \mathrm{C}$ for $24 \mathrm{~h}$. Growth within $5 \mathrm{~mm}$ of the margin of the disc was considered potentially significant.

2) Selective ChromID ESBL-agar (Biomerieux, France) and Gentamicin-MacConkey plates. The latter was prepared in-house by adding $800 \mu \mathrm{l}$ Gentamicin $10 \mathrm{mg} / \mathrm{ml}$ (Gibco, UK) in $1 \mathrm{~L}$ of MacConkey agar without salt. Any growth on these plates was considered potentially significant.

3) Samples were selectively enriched overnight in $5 \mathrm{ml}$ nutrient broth with either a Cefpodoxime $(10 \mu \mathrm{g})$ or a Gentamicin $(10 \mu \mathrm{g})$ disc added to the bijou bottle. These enriched samples were sub-cultured the following day and then handled in the same way as the direct methods above.

Where results were discordant between different sample types from the same patient using the screening methods, any detection of a resistant organism was considered to be the correct identification. A single colony of each distinguishable type (up to three per sample) was picked from a resistance screening plate, grown on a purity plate and subsequently tested as described below.

\section{Identification of isolates}

Bacterial isolates expressing either ESBL or Gentamicin resistance or both were obtained. An oxidase test was performed (Remel Bactidrop, UK) for all isolates. Oxidasenegative isolates were identified to the family/genus level using API10S kits (Biomerieux, France) according to the manufacturer's instructions; this system does not consistently provide identification to the species level. The ESBL phenotype was confirmed by paired disc tests [18] according to the manufacturer's instructions (Mast Diagnostics, UK). Where a sample had no isolates confirmed as ESBL producers by paired disc testing, we considered the sample to be negative for that type of resistance throughout the analysis.

\section{Antimicrobial susceptibility testing}

Overnight pure cultures confirmed to be ESBL positive or gentamicin resistant had further susceptibility testing 
performed using the standard disc diffusion method based on Clinical Laboratory Standards Institute (CLSI) breakpoints for susceptibility testing. The antibiotics tested were gentamicin $(10 \mu \mathrm{g})$, chloramphenicol $(30 \mu \mathrm{g})$, ciprofloxacin $(1 \mu \mathrm{g})$, ertapenem $(10 \mu \mathrm{g})$, cefpodoxime $(10 \mu \mathrm{g})$ and ampicillin (10 $\mu \mathrm{g})$ (Oxoid, England). Reference strains E. coli ATCC25922 (fully susceptible) and E. coli NCTC13353 (ESBL CTX-M type and gentamicin resistant) were used as control organisms. Data were recorded in a Microsoft Access database and STATA v13 was used to calculate prevalence and acquisition rates of resistance carriage and perform tests of association.

\section{Results}

\section{Admissions}

A total of 164 paediatric inpatients were recruited to the study out of a total of 1757 paediatric admissions to these two hospitals in the study period. Most patients $(142 / 164,87 \%)$ were recruited on the day following their admission to hospital (hence after spending one night in hospital), but all within $24 \mathrm{~h}$ of their admission. A summary of these patients is shown in Table 1 . The median age was 1.0 years (IQR 0.2 to 2.2 years). The most commonly recorded admission diagnoses were "severe pneumonia", "sepsis" and "severe malnutrition". The average duration of hospitalization was 5.6 days (IQR 2-7.5 days;

Table 1 Summary of study admissions

\begin{tabular}{lcc}
\hline & Number $(n=164)$ & $\%$ \\
\hline Age of child & 19 & $12 \%$ \\
$0-28$ days & 63 & $38 \%$ \\
29 days-1 year & 64 & $39 \%$ \\
$1-5$ years & 18 & $11 \%$ \\
Over 5 years & & \\
Ward of recruitment & 41 & $25 \%$ \\
Harare Childrens Hospital, ward B1 & 63 & $38 \%$ \\
Harare Childrens Hospital, ward B2 & 3 & $3 \%$ \\
Parirenyatwa Hospital, admission unit & 22 & $22 \%$ \\
Parirenyatwa Hospital, ward A4 General & 24 & $24 \%$ \\
Parirenyatwa Hospital, ward A4 Special & 11 & $11 \%$ \\
Parirenyatwa Hospital, ward A5 & & \\
Enteric carriage of resistance on admission & 68 & $41 \%$ \\
Both ESBL and gentamicin resistance & 18 & $11 \%$ \\
ESBL resistance only & 17 & $10 \%$ \\
Gentamicin resistance only & 61 & $37 \%$ \\
Neither form of resistance & & \\
Outcome of admission & 151 & $92 \%$ \\
Discharged & 13 & \\
Died & & \\
\hline
\end{tabular}

range 1-32 days) and the inpatient mortality was $8 \%$ (13 deaths/164 admissions).

\section{Carriage of antibiotic resistance on admission}

The prevalence of ESBL carriage was 52\% (86/164) and gentamicin resistance carriage was 52\% (85/164), on the initial sample taken from patients, with $41 \%$ of all patients having both forms of resistance. On admission, ESBL carriage was higher amongst children in the age groups 29 days-1 year $(39 / 63 ; 62 \%)$ and $1-5$ years $(37 / 64 ; 58 \%)$ and lower in the younger $(0-28$ days $(7 / 19 ; 37 \%))$ and older $(5+$ years $(3 / 18 ; 18 \%)$ age groups; (Fishers exact test across these categories $p=0.001$ ). We found no difference in carriage rates by age group for gentamicin resistance on admission to hospital. Only $37 \%$ of children had neither form of resistance detectable-see Table 1 . There was no association between hospital of admission and prevalence of carriage of ESBL ( $\chi^{2}$-test $p=0.23$ ) or gentamicin resistance $(p=0.32)$. Death as an inpatient was associated with ESBL carriage on admission (Fisher's exact test $p=0.02$ ) but not with gentamicin resistance $(p=0.25)$.

\section{Acquisition of carriage during admission}

An average of 1.9 samples were obtained per patient (range $=1$ to 8 ) with 91 patients having two or more samples collected. A total of 32 admissions were detected to have a new form of resistance during their hospital admission: 17 gained both ESBL and gentamicinresistance, 12 gained ESBL only and 3 gained gentamicin resistance only (see Table 2). There was no association between hospital and acquisition of resistance during admission for either ESBL $\left(\chi^{2}\right.$-test $\left.p=0.40\right)$ or gentamicin resistance $\left(\chi^{2}\right.$-test $\left.p=0.40\right)$. The probability of any sample containing a resistant organism was closely related to the time since admission to hospital-see Fig. 1. After a patient had been hospitalised for five or more days, there was over $90 \%$ probability (86/93 samples) that carriage of both ESBL and gentamicin-resistant organisms would be detected in a faecal specimen.

\section{Screening methods for detection of resistant organisms}

A total of 312 samples were collected, of these 143 were rectal swabs only, 23 were faecal samples only and 146 had both types of specimen. In the samples with both rectal swabs and faecal samples, the sensitivity of the

Table 2 Summary of ESBL or Gentamicin resistance acquisition status during admissions

\begin{tabular}{ll}
\hline$>1$ faecal sample taken during hospital admission & $91 / 164(55 \%)$ \\
New detection of ESBL during admission & $29 / 164(18 \%)$ \\
New detection of gentamicin resistance during admission & $20 / 164(12 \%)$ \\
New detection of either form of resistance & $32 / 164(20 \%)$ \\
New detection of both forms of resistance & $17 / 164(10 \%)$ \\
\hline
\end{tabular}




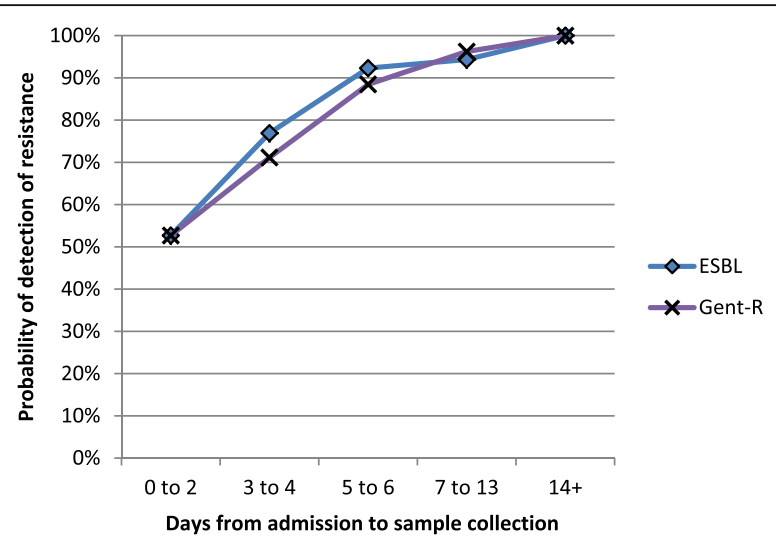

Fig. 1 Probability of detection $v$ time-to-sample

different detection techniques is shown in Table 3 . Broadly speaking, we found that higher sensitivity was achieved with faecal samples and inclusion of the enrichment broth step. Use of selective media did not achieve substantially higher sensitivity, but was noted to be practically easier to use in the laboratory. Out of the oxidasenegative Gram-negative isolates detected by the ESBL screening methods and confirmed as Enterobacteriaceae, the large majority $(190 / 214 ; 89 \%)$ were confirmed as ESBL by both disc pairs (cefotaxime/clavulanate pair and ceftazidime/clavulanate pair) and some were confirmed by only a single pair of ceftazidime/clavulanate (16/214; 7.5\%). A small number of isolates were not confirmed as ESBLproducers by either disc pair $(8 / 214 ; 4 \%)$.

\section{Antibiotic resistance profiles}

Amongst the isolated Enterobacteriaceae that were confirmed ESBL $(n=206)$ or gentamicin resistant $(n=146)$, we examined the co-resistance to other commonly used antibiotics-see Tables 4 and 5. We found high levels of co-resistance to ampicillin, ciprofloxacin and chloramphenicol, but virtually no co-resistance to ertapenem.

\section{Discussion}

Our study investigated enteric carriage of ESBLproducing and gentamicin resistant enteric bacteria in a sample of 164 hospitalised children drawn from two tertiary public-sector hospitals in Harare, Zimbabwe over a 3 month period. The main findings were high rates of carriage of ESBL and gentamicin resistant bacteria on admission to hospital and high rates of acquisition for the same resistance types during the inpatient period.

An initial sample was collected within $24 \mathrm{~h}$ of hospital admission for each patient, so these samples are likely to represent the carriage status prior to the current hospital admission. Other studies of ESBL prevalence around the time of admission to hospital have found carriage rates of 33\% (133/408) in Guinea Bissau [9], 34\% (37/110) in Gabon [12] and 22\% (54/244) in Madagascar [11]. Amongst neonates in Tanzania, 25\% (32/126) were found to have ESBL carriage, predominantly Klebsiella pneumoniae [13]. In a study conducted in a renutrition centre in Niger [10], ESBL carriage was 31\% (17/55 patients) on admission and by time of discharge a further 15/16 children had acquired carriage. Our study, showed a higher prevalence of carriage than those previously described levels, though our methods of screening were probably more sensitive than the direct plating of rectal swabs used elsewhere. We are not aware of any previous African studies specifically investigating carriage of gentamicin resistance in hospitalised children. Carriage of resistance amongst hospital admissions is liable to be higher than carriage in the general community, as sick individuals probably have exposure to various healthcarerelated risk factors prior to admission, so we cannot compare these results to truly community-based studies. However, the extremely high levels of carriage rates found suggest that circulation of ESBL and gentamicin resistance probably occurs in the community in Harare. Although we found an association between ESBL carriage and inpatient death, we were not able to assess for the confounding effects and hence it remains uncertain whether this is a causal association.

Collection of multiple follow-up samples during the inpatient period allowed us to observe acquisition of carriage of resistance over time. An important question is whether these in-hospital acquisitions are occurring via endogenous (arising from undetectably low levels already present in the gastrointestinal tract) or exogenous (arising

Table 3 Sensitivity of screening methods for resistant organisms ( $n=146$ samples)

\begin{tabular}{|c|c|c|c|c|}
\hline \multirow[b]{2}{*}{ Sample type } & \multicolumn{2}{|l|}{ ESBL } & \multicolumn{2}{|c|}{ Gentamicin-resistant } \\
\hline & Rectal swab & Faecal sample & Rectal swab & Faecal sample \\
\hline Plate with disc & $73 \%$ & $84 \%$ & $66 \%$ & $83 \%$ \\
\hline Selective plate & $75 \%$ & $87 \%$ & $70 \%$ & $82 \%$ \\
\hline $\begin{array}{l}\text { Enrichment broth } \\
\text { THEN plate with disc }\end{array}$ & $90 \%$ & $96 \%$ & $87 \%$ & $95 \%$ \\
\hline $\begin{array}{l}\text { Enrichment broth } \\
\text { THEN selective plate }\end{array}$ & $90 \%$ & $96 \%$ & $84 \%$ & $96 \%$ \\
\hline
\end{tabular}


Table 4 Susceptibility profiles of confirmed ESBL-producing isolates $(n=206)$

\begin{tabular}{lccc}
\hline Antibiotic & Number resistant & \% Resistant & Total tested \\
\hline Gentamicin & 130 & 65 & 199 \\
Ampicillin & 199 & 100 & 199 \\
Ciprofloxacin & 162 & 82 & 198 \\
Chloramphenicol & 105 & 53 & 198 \\
Ertapenem & 2 & 1 & 199 \\
\hline
\end{tabular}

from cross-infection from an external source) routes. This distinction has important implications for antibiotic stewardship and infection control measures. For endogenous routes of acquisition, antibiotic stewardship activities focussing on reducing the selective pressure from antibiotic prescribing are important. For exogenous acquisition, measures to minimise cross-infection, such as improved hand hygiene, are priorities. Whilst we cannot be certain, we feel that both routes are likely to be contributing to the high levels of nosocomial acquisition seen here. Our screening methods included an enrichment step so that we should have been able to detect small quantities of resistant bacteria, making endogenous acquisition less likely, though a single faecal specimen is not a representative sample of the estimated $10^{14}$ cells in the intestinal microbiome. Enrichment broths with supplementary antibiotics have been used elsewhere to aid detection of resistant organisms in faecal samples [18]. Our clinical experience in these hospitals suggests there are substantial challenges with over-crowding, limited access to hand-hygiene facilities and high patient:staff ratios. With regard to handhygiene, at the time the study was conducted, water supplies in these wards were sporadic and alcohol-based hand rub (ABHR) was not widely available. Concurrent work conducted in the same wards as a separate part of this pilot study showed that hands of adult carers were frequently contaminated with Gram-negative antibiotic resistant bacteria [19]. These are all factors that might be expected to contribute to exogenous routes of transmission.

In line with findings from other studies, we found that most ESBL-producing bacteria from these children were also resistant to several other clinically-relevant antibiotic

Table 5 Susceptibility profiles of Gentamicin resistant isolates $(n=146)$

\begin{tabular}{lccc}
\hline Antibiotic & Number resistant & \% Resistant & Total tested \\
\hline Cefpodoxime $^{a}$ & 126 & 86 & 146 \\
Ampicillin & 144 & 99 & 146 \\
Ciprofloxacin & 112 & 77 & 146 \\
Chloramphenicol & 77 & 53 & 146 \\
Ertapenem & 0 & 0 & 145 \\
\hline
\end{tabular}

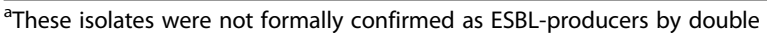
disc testing classes [9-13]. This represents a substantial clinical challenge for empirical treatment of suspected hospitalacquired infections-ciprofloxacin (for severe urinary sepsis) and chloramphenicol (for bacteraemia and meningitis) represent important and widely used second-line treatment options in many African hospitals. The level of carbapenem resistance amongst resistant isolates was very low, but as these agents are not currently routinely available through public-sector pharmacies in Zimbabwe, this information is of limited practical value for local clinicians.

Due to rapid turnover of patients, we were only able to collect follow-up samples in a moderate number of patients; 73 patients had only a single sample collected. Whilst this does not affect estimates for the time of admission, it limits the ability of the study to detect new acquisition of carriage-in reality, probably even more children became carriers of resistance during admission than we detected. Ideally, an additional sample on the day of discharge would have been collected for all patients. Our simple screening and identification methods meant that we could not distinguish the simultaneous carriage of multiple different resistant organisms. Again, this means that the acquisition risk described here may underestimate the true in-hospital acquisition risk. We did not make use of molecular techniques for identification of resistance mechanisms or for investigating transmission of resistance. In this preliminary study, we were not able to evaluate clinical impacts of antibiotic resistance, either during hospital inpatient stay or after discharge. Whilst we did find some evidence that carriage of ESBL resistance was associated with inpatient mortality, investigating whether carriage of resistance was followed by clinical infection with the same organisms was beyond the scope of the study.

Future research is needed to better understand and tackle these high levels of antibiotic resistance. This will take many forms. We did not collect information on risk factors associated with carriage of resistance-this would be a valuable first step in understanding why this is occurring. In other settings, prior use of antibiotics and recent hospital admission have been associated with carriage of resistance [20] - we do not know if this is the case in Zimbabwe. More work is needed to identify the enzyme types and genes responsible for the forms of resistance that we detected-this will enable us to start to understand the dynamics of resistance transmission. Screening for drug-resistance on admission and cohorting or isolating patients can result in improved patient outcomes [21]_but no modelling work has ever been conducted to explore the value of this in the setting of African hospitals. We do not know how the acquisition of antibiotic resistance in hospitals relates to resistance in the community in Zimbabwe [22], nor do we know 
the wider impact of antibiotic resistance in terms of mortality, morbidity and costs in sub-Saharan African countries, though we suspect these to be substantial [23]. Given that many African hospitals have limited access to carbapenems, infections caused by ESBL-producers might have a substantially greater impact than has been seen in high-income settings [8].

At a more practical level, immediate action can be taken in a number of areas such as improving infection control practices, especially hand hygiene, which has been shown to reduce infections caused by antibiotic-resistant Gramnegative bacteria in high-income settings [24]. The use of alcohol-based hand rub as part of the WHO 5 moments for hand hygiene approach is a low-cost technology that could be much more widely adopted in African hospitals. Ideally, a rigorous evaluation of the implementation of such new practices would contribute to the evidence-base for these measures in low-income settings, which is currently extremely limited [25].

\section{Conclusion}

The rising level of antimicrobial resistance is of great concern worldwide. These high rates of ESBL and gentamicin resistance carriage found amongst paediatric inpatients in Zimbabwe are particularly worrisome. These bacteria pose risks of multi-drug resistant infections and transmission to other hospital inpatients. The high levels of carriage at the time of hospital admissions suggest that they are already in widespread circulation in the general community. Relevant infection control measures such as access to alcohol-based hand rub should be urgently scaled up in these hospitals and beyond, so that children who arrive at hospital without carriage of antibiotic resistant bacteria can remain free of these for the duration of their admissions.

\section{Abbreviations}

ABHR: Alcohol-based hand rub; CLSI: Clinical Laboratory Standards Institute; ESBL: Extended- spectrum $\beta$-lactamase; IQR: Inter-quartile range

\section{Acknowledgements \\ We are grateful to the study nurses Dorah Kaisi and Phillipa Dingiswayo from the Department of Paediatrics and Child Health UZ for their diligent work on recruitment of patients and collection of samples. We would like to thank patients, parents and staff in the participating wards for their assistance with this work. We thank staff in the Department of Medical Microbiology, University of Zimbabwe and the Biomedical Research and Training Institute for their support.}

\section{Funding}

This work was funded by an Academy of Medical Sciences Starter Grant for Clinical Lecturers (AA, grant reference AMS-SGCL11-AikenA). This work formed part of a pilot study ahead of a cluster-randomised trial of a hand-hygiene intervention in hospitals in sub-Saharan Africa.

\section{Availability of data and materials}

The dataset and files showing the calculations in the papers are available from the following link. https://dl.dropboxusercontent.com/u/69222597/ ESBL\%20paper\%20data\%2Bdo\%20files.zip.

\section{Authors' contributions}

Conceived and designed the experiments: MM, MGM, HAM, MBD, VR, AA. Performed the experiments: MM MGM. Analysed the data: MM AA. Contributed reagents/materials/analysis tools: VR AA. Wrote the manuscript: MM AA.

All authors read and approved the manuscript.

\section{Competing interests}

The authors declare that they have no competing interests.

\section{Consent for publication}

Not applicable-the manuscript does not include details, images, or videos relating to individual participants but rather presents analysis of aggregated data on the study population.

\section{Ethics approval and consent to participate}

The Institutional Review Boards of both hospitals, the Medical Research Council of Zimbabwe and the London School of Hygiene and Tropical Medicine approved the study. Parents or guardians of all participants gave written informed consent to participate in the study.

\section{Author details}

${ }^{1}$ College of Health Sciences, Department of Medical Microbiology, University of Zimbabwe, PO Box A178, Harare, Zimbabwe. ${ }^{2}$ National Microbiology Reference Laboratory, Harare Central Hospital, Harare, Zimbabwe. ${ }^{3}$ College of Health Sciences, Department of Paediatrics and Child Health, University of Zimbabwe, Harare, Zimbabwe. ${ }^{4}$ Harare Central Hospital, Harare, Zimbabwe. ${ }^{5}$ Parirenyatwa Group of Hospitals, Harare, Zimbabwe. ${ }^{6}$ Biomedical Research and Training Institute, Harare, Zimbabwe. 'London School of Hygiene and Tropical Medicine, London, UK.

Received: 19 July 2016 Accepted: 26 November 2016 Published online: 11 January 2017

\section{References}

1. WHO. Worldwide country stiuation analysis: response to antimicrobial resistance. 2015

2. Ma X, Wu Y, Li L, Xu Q, Hu B, Ni Y, et al. First multicenter study on multidrug resistant bacteria carriage in Chinese ICUs. BMC Infect Dis. 2015;15:358.

3. Yong D, Toleman MA, Giske CG, Cho HS, Sundman K, Lee K, et al. Characterization of a new metallo-beta-lactamase gene, bla(NDM-1), and a novel erythromycin esterase gene carried on a unique genetic structure in Klebsiella pneumoniae sequence type 14 from India. Antimicrob Agents Chemother. 2009;53(12):5046-54.

4. Carrer A, Poirel L, Yilmaz M, Akan OA, Feriha C, Cuzon G, et al. Spread of OXA-48-encoding plasmid in Turkey and beyond. Antimicrob Agents Chemother. 2010;54(3):1369-73.

5. Allegranzi B, Bagheri Nejad S, Combescure C, Graafmans W, Attar H, Donaldson $L$, et al. Burden of endemic health-care-associated infection in developing countries: systematic review and meta-analysis. Lancet. 2011;377(9761):228-41.

6. Thaden JT, Fowler VG, Sexton DJ, Anderson DJ. Increasing incidence of extended-spectrum beta-lactamase-producing Escherichia coli in community hospitals throughout the Southeastern United States. Infect Control Hosp Epidemiol. 2016;37(1):49-54.

7. Lukac PJ, Bonomo RA, Logan LK. Extended-spectrum beta-lactamaseproducing enterobacteriaceae in children: old foe, emerging threat. Clin Infect Dis. 2015:60(9):1389-97.

8. de Kraker ME, Wolkewitz M, Davey PG, Koller W, Berger J, Nagler J, et al. Burden of antimicrobial resistance in European hospitals: excess mortality and length of hospital stay associated with bloodstream infections due to Escherichia coli resistant to third-generation cephalosporins. J Antimicrob Chemother. 2011;66(2):398-407.

9. Isendahl J, Turlej-Rogacka A, Manjuba C, Rodrigues A, Giske CG, Naucler P. Fecal carriage of ESBL-producing E. coli and K. pneumoniae in children in Guinea-Bissau: a hospital-based cross-sectional study. PLoS One. 2012;7(12):e51981.

10. Woerther $\mathrm{PL}$, Angebault $\mathrm{C}$, Jacquier $\mathrm{H}$, Hugede $H C$, Janssens $A C$, Sayadi $\mathrm{S}$, et al. Massive increase, spread, and exchange of extended spectrum beta-lactamase-encoding genes among intestinal Enterobacteriaceae in 
hospitalized children with severe acute malnutrition in Niger. Clin Infect Dis. 2011:53(7):677-85.

11. Andriatahina T, Randrianirina F, Hariniana ER, Talarmin A, Raobijaona $H$, Buisson $Y$, et al. High prevalence of fecal carriage of extended-spectrum beta-lactamase-producing Escherichia coli and Klebsiella pneumoniae in a pediatric unit in Madagascar. BMC Infect Dis. 2010;10:204.

12. Schaumburg F, Alabi A, Kokou C, Grobusch MP, Kock R, Kaba H, et al. High burden of extended-spectrum beta-lactamase-producing

Enterobacteriaceae in Gabon. J Antimicrob Chemother. 2013;68(9):2140-3.

13. Nelson E, Kayega J, Seni J, Mushi MF, Kidenya BR, Hokororo A, et al.

Evaluation of existence and transmission of extended spectrum beta lactamase producing bacteria from post-delivery women to neonates at Bugando Medical Center. Mwanza-Tanzania BMC Res Notes. 2014;7:279.

14. Chen C, Chen Y, Wu P, Chen B. Update on new medicinal applications of gentamicin: evidence-based review. J Formos Med Assoc. 2014;113(2):72-82.

15. Carlet J. The gut is the epicentre of antibiotic resistance. Antimicrob Resist Infect Control. 2012;1(1):39.

16. Aiken AM, Mturi N, Njuguna P, Mohammed S, Berkley JA, Mwangi I, et al. Risk and causes of paediatric hospital-acquired bacteraemia in Kilifi District Hospital, Kenya: a prospective cohort study. Lancet. 2011;378(9808):2021-7.

17. Donskey CJ. The role of the intestinal tract as a reservoir and source for transmission of nosocomial pathogens. Clin Infect Dis. 2004;39(2):219-26.

18. Thomson KS. Extended-spectrum-beta-lactamase, AmpC, and Carbapenemase issues.J Clin Microbiol. 2010:48(4):1019-25.

19. Gudza-Mugabe M, Magwenzi MT, Mujuru HA, Bwakura-Dangarembizi M, Robertson V, Aiken AM. Decontamination of hands using locallymanufactured alcohol-based hand rub in paediatric wards in Harare, Zimbabwe. Antimicrob Resist Infect Control, in press.

20. Pop-Vicas AE, D'Agata EM. The rising influx of multidrug-resistant gram-negative bacilli into a tertiary care hospital. Clin Infect Dis. 2005:40(12):1792-8.

21. Tacconelli E, Cataldo MA, Dancer SJ, De Angelis G, Falcone M, Frank U, et al. ESCMID guidelines for the management of the infection control measures to reduce transmission of multidrug-resistant Gram-negative bacteria in hospitalized patients. Clin Microbiol Infect. 2014;20 Suppl 1:1-55.

22. Hilty M, Betsch BY, Bogli-Stuber K, Heiniger N, Stadler M, Kuffer M, et al. Transmission dynamics of extended-spectrum beta-lactamase-producing Enterobacteriaceae in the tertiary care hospital and the household setting. Clin Infect Dis. 2012;55(7):967-75.

23. Aiken AM, Allegranzi B, Scott JAG, Mehtar S, Pittet D, Grundmann H. Letter responding to "Antibiotic resistance - the need for Global Solutions" by Laxminarayan et al. Lancet Infect Dis. 2014;14(July):550-1.

24. Johnson PD, Martin R, Burrell $\amalg$, Grabsch EA, Kirsa SW, O'Keeffe J, et al. Efficacy of an alcohol/chlorhexidine hand hygiene program in a hospital with high rates of nosocomial methicillin-resistant Staphylococcus aureus (MRSA) infection. Med J Aust. 2005;183(10):509-14.

25. Luangasanatip N, Hongsuwan M, Limmathurotsakul D, Lubell Y, Lee AS, Harbarth $\mathrm{S}$, et al. Comparative efficacy of interventions to promote hand hygiene in hospital: systematic review and network meta-analysis. BMJ. 2015;351:h3728.

\section{Submit your next manuscript to BioMed Central and we will help you at every step:}

- We accept pre-submission inquiries

- Our selector tool helps you to find the most relevant journal

- We provide round the clock customer support

- Convenient online submission

- Thorough peer review

- Inclusion in PubMed and all major indexing services

- Maximum visibility for your research

Submit your manuscript at www.biomedcentral.com/submit

) Biomed Central 